\title{
Estratégias de avaliação de condutores de automóveis com mobilidade reduzida
}

\section{Strategies to evaluate drivers with reduced mobility}

\author{
Andrea Maria Fedeger ${ }^{1}$, Helio Augusto Ghizoni Teive ${ }^{2}$, Renato Nickel ${ }^{1}$
}

http://dx.doi.org/10.11606/issn.2238-6149.v29i1p50-55

Fedeger AM, Teive AG, Nickel R. Estratégias de avaliação de condutores de automóveis com mobilidade reduzida. Rev Ter Ocup Univ São Paulo. 2018 jan.-abr.;29(1):50-5.

RESUMO: Este estudo tem como objetivo apresentar estratégias para avaliação de condutores de automóveis, com mobilidade reduzida, para terapeutas ocupacionais. Entre as atividades instrumentais da vida diária (AIVD), a mobilidade na comunidade, especificamente dirigindo um carro, é uma atividade que promove autonomia, bemestar e participação na comunidade. A aptidão para conduzir é o motorista que integra habilidades motoras, visuais e cognitivas dinâmicas. Além disso, o motorista precisa demonstrar conhecimento na legislação de trânsito, controlar o veículo e ter validade na Licença de Motorista Nacional (CNH). Os dados foram produzidos entre 2011 e 2012 através de revisão bibliográfica, documental e pesquisa empírica. Na pesquisa bibliográfica foi utilizada a base de dados LILACs. A legislação brasileira de transito contextualizou as normas neste país. O descritivo transversal é parte da pesquisa chamada "Um estudo descritivo do desempenho de veículos a motor em pessoas diagnosticadas com doença de Parkinson". Um questionário estruturado na língua inglesa foi aplicado para dois terapeutas ocupacionais norte-americanos. $\mathrm{O}$ estudo apresentou a descrição do terapeuta ocupacional como reabilitação do motorista. Foi possível descrever os procedimentos de avaliação de condutores realizados pelo terapeuta ocupacional, incluindo seu campo de prática e desempenho esperado para dirigir um veículo.

DESCRITORES: Terapia ocupacional; Condução de veículo; Estudos de avaliação como assunto; Avaliação da deficiência.
Fedeger AM, Teive AG, Nickel R. Strategies to evaluate drivers with reduced mobility. Rev Ter Ocup Univ São Paulo. 2018 Jan.-Apr.;29(1):50-5.

ABSTRACT: This study aims to present strategies to evaluation drivers with reduced mobility for occupational therapists. Among the Instrumental Activities of Daily Life (AIVD), mobility in the community, specifically driving a car, is an activity that promotes autonomy, well-being and participation in the community. Fitness to drive is the driver who integrates dynamically motor, visual and cognitive skills. Also the driver needs demonstrate knowledge in traffic legislation, control the vehicle and have validity in the National Driver's License $(\mathrm{CNH})$. The data were produced between 2011 and 2012 through bibliographic, documental review and empiric research. The databases was LILACs. The Brazilian transit legislation has contextualized the norms in this country. The cross-sectional descriptive is part of the research called "A descriptive study of the performance of motor vehicles in people diagnosed with Parkinson's disease". A structured questionnaire in the English language was applied for two North American occupational therapists. The study presented the description of the occupational therapist as driver rehabilitation. There are description assessment procedures the occupational therapists including their practice field and expected performance to drive a vehicle.

KEYWORDS: Occupational therapy; Automobile driving; Evaluation studies as topic; Disability evaluation.

\footnotetext{
Este trabalho é parte integrante da Tese de Doutorado intitulada "Avaliação de Condutores de Automóveis com Doença de Parkinson: um estudo em Curitiba/PR" e do Projeto de Pesquisa "Estudo descritivo do desempenho na condução de automóveis de pessoas com o diagnóstico da Doença de Parkinson".

1. Docente do Curso de Graduação em Terapia Ocupacional, Departamento de Terapia Ocupacional, Universidade Federal do Paraná.

E-mail: andrea.fedeger@gmail.com,renatonickel@hotmail.com

2. Docente do Departamento de Neurologia da Universidade Federal do Paraná. E-mail: teiveads@mps.com.br Endereço para correspondência: Rua Raquel Prado, 1247/1. Bom Retiro. Curitiba, PR, BR. CEP: 80520290.
} 


\section{INTRODUÇÃO}

mobilidade resulta na movimentação
$\begin{aligned} & \text { corpórea funcional dos segmentos } \\ & \text { anatômicos, com ou sem uso de tecnologia }\end{aligned}$ assistiva. A mobilidade, uma atividade instrumental de vida diária (AIVD) promove a emancipação e participação das pessoas na comunidade, podendo ser desempenhada pelo uso de transportes públicos e privados, por exemplo a condução de um veículo ${ }^{1,2,3}$.

Este estudo tem como foco a condução de automóveis de pessoas com mobilidade reduzida, entendida a partir do Código de Trânsito Brasileiro (CTB) e da Norma Brasileira de Acessibilidade (NBR 9050) como a "pessoa que tem limitada, temporária ou permanentemente, sua capacidade de relacionar-se com o meio e de utilizá-lo, [...] a pessoa com deficiência, idosa, obesa, gestante entre outros" (p.4) ${ }^{4}$. A garantia das pessoas com mobilidade reduzida do direito de ir e vir conduzindo um automóvel está descrita em normativa e inclui os comandos e requisitos de dirigibilidade ${ }^{4}$.

Para conduzir, o condutor apto e com privilégio da Carteira Nacional de Habilitação (CNH) deve estar em posição sentada no automóvel e manter-se assim durante o tempo necessário para percorrer o trajeto. Ao desempenhar essa atividade, todo condutor deve garantir que nenhuma parte do seu corpo acione indevidamente algum comando do veículo. O CTB, órgão normativo do trânsito, imputa infrações, multas e a suspensão da $\mathrm{CNH}$ ao condutor de risco. O risco é resultado de atitudes de desrespeito às normas e sinalizações das vias de circulação ou pelo desempenho ineficiente, inabilidade física ou mental, em manobras perigosas sem controle do veículo na via à pedestres e outros motoristas no trânsito ${ }^{5}$.

Conduzir um automóvel é uma atividade complexa e de risco que compõe a rotina de muitas pessoas. No Brasil, há 60 milhões de motoristas com $\mathrm{CNH}$ válida ${ }^{6}$, entretanto, não há no país informações acerca do número de pessoas com mobilidade reduzida que possuem $\mathrm{CNH}$ válida. Vivemos uma década denominada pela Assembleia Geral das Nações Unidas como Década de Ação para a Segurança Rodoviária (2011-2020) que tem como metas a redução do número de mortes e lesões e implementação de programas de atenção à saúde que promovam a segurança no trânsito ${ }^{6}$. Os acidentes de trânsito no Brasil, em 2011, segundo o Sistema de Informações sobre Mortalidade (SIM), totalizaram em 43.256 óbitos. Entende-se que os acidentes de trânsito são evitáveis, já que em todo acidente de trânsito sempre está presente uma falha humana ${ }^{7,89}$.
No Brasil, o processo de avaliação de condutores de automóveis, com mobilidade reduzida ou não, desde a concessão e a renovação da $\mathrm{CNH}$ está descrita na Política Nacional de Trânsito (PNT) e nas resoluções do CBT. Segundo a Resolução $\mathrm{n}^{\circ} 267$, de 15 de fevereiro de $2008^{10}$, os agentes responsáveis pela avaliação do desempenho do condutor de veículos são: médico perito em trânsito e o psicólogo perito em trânsito. Além desses profissionais há o instrutor de trânsito, o qual, segundo a Lei $n^{\circ} 12.302$, de 2 de agosto de $2010^{11}$, responsabiliza-se pela formação do futuro condutor e o orienta durante a aprendizagem da direção veicular. A especialidade do terapeuta ocupacional como reabilitador de condutores de automóveis é realidade nos Estados Unidos da América ${ }^{2,8}$.

A partir de diretrizes para terapeutas ocupacionais na reabilitação de condutores de automóveis da Associação Americana de Terapia Ocupacional (AOTA) ${ }^{12}$, este estudo objetiva apresentar estratégias para avaliação de condutores de automóveis, com mobilidade reduzida, para terapeutas ocupacionais.

Para o terapeuta ocupacional brasileiro, essa temática emerge como uma nova possibilidade de prática profissional que requer conhecimento específico e ético para atuar na reabilitação de condutores de automóveis. Esta pesquisa, vinculado à capacitação profissional, aponta horizontes para a produção do conhecimento em novo cenário de prática profissional inexistente no Brasil e implica a necessidade de interlocução com politicas publicas voltadas ao desenvolvimento das cidades e da segurança no transito.

\section{PROCEDIMENTOS METODOLÓGICOS}

Este trabalho é um recorte do projeto de pesquisa intitulado "Estudo descritivo do desempenho na condução de automóveis de pessoas com doença de Parkinson”. Esta pesquisa foi aprovada pelo Comitê de Ética em Pesquisa em Seres Humanos do Setor de Ciências da Saúde (SD) Plataforma Brasil (PB) / Universidade Federal do Paraná (UFPR) em 26 outubro de 2011, com registro de número 675558 / SD-PB CEP.

Para este estudo foram realizadas revisão bibliográfica, pesquisa documental e pesquisa de campo. A revisão bibliográfica buscou em bases de dados na Literatura Latino-Americana e do Caribe de Ciências da Saúde (LILACS) com publicações entre 2000 e 2011. $\mathrm{Na}$ pesquisa documental foram realizadas leituras em documentos e arquivos de órgãos públicos relacionados a PNT e ao CBT, em sua maioria as leis, portarias e resoluções. 
O estudo descritivo do desempenho de condutores com Doença de Parkinson teve início em 2011. A revisão de literatura precedeu a visita técnica. Em Janeiro de 2012, realizou-se a visita técnica ao Institute for Mobility, Activity, \& Participation (I-MAP). O I-MAP está vinculado ao College of Public Health and Health Professions da Universidade da Florida em Gainesville, Florida, Estados Unidos da América. Com duração aproximada de 6 horas, a visita foi conduzida pela Professora Doutora Sherrilene Classen. O conhecimento produzido neste importante acontecimento na pesquisa motivou a construção do questionário no idioma inglês para conhecer mais profundamente a prática do terapeuta ocupacional. O questionário com 16 questões fechadas investigou o perfil e a formação do terapeuta ocupacional, os procedimentos de avaliação na reabilitação de condutores de automóveis com mobilidade reduzida. Para responder ao questionário, estimou-se o tempo aproximado de 30 minutos.

Após contato prévio, em Maio de 2012, o questionário foi enviado por email para duas terapeutas ocupacionais especialistas em reabilitação de condutores, juntamente com o Termo de Consentimento Livre e Esclarecido (TCLE). O critério de inclusão na seleção de profissionais foi por escolha de profissionais vinculadas no I-MAP. O prazo de retorno das respostas e do TCLE assinado foi de cinco dias úteis. Após o recebimento dos dados produzidos pelas duas profissionais, os mesmos foram registrados em tabela Excel, a partir da qual foi realizada análise e discussão descritiva dos resultados.

\section{RESULTADOS E DISCUSSÃO}

Os dados produzidos no questionário $\mathrm{e}$ as contribuições bibliográficas realizados nesta pesquisa serão delineados considerando a formação e o lugar do terapeuta ocupacional e procedimentos técnicos no processo de reabilitação de condutores de automóveis.

Sobre a formação e o lugar do terapeuta ocupacional na reabilitação de condutores de automóveis serão apresentados o processo de certificação e papéis do profissional, espaços de atuação e raciocínio profissional sobre a atividade.

A média de 15 anos de formação em Terapia Ocupacional as duas profissionais confirmaram o vinculo com o I-MAP. Para preservar o sigilo, as participantes foram denominadas TOA e TOB. As duas terapeutas ocupacionais são especialistas certificadas em reabilitação de condutores (Certified Driver Rehabilitation Specialist - CDRS) em média há 5 anos pela Association for Driver Rehabilitation Specialist (ADED). As profissionais descreveram sobre educação permanente para desempenhar tal função. TOA relatou que cursou formação específica na cidade de Orlando, Flórida, recebeu treinamento da terapeuta ocupacional Wendy Stav, que é uma referência nesta especialidade e há 8 anos prestou exame para ser certificada como especialista em reabilitação de condutores. A TOB informou que participou de curso presencial e online e há 7 anos prestou exame onde obteve certificação como especialista em reabilitação de condutores. As duas profissionais relataram que continuamente atualizam-se para a prática em leitura de livros e artigos e participação de eventos e cursos nesta especialidade.

As participantes descreveram que no I-MAP atendem condutores com e sem mobilidade reduzida, sendo pessoas com queixas cognitivas, visuais e/ou motoras associadas a outras condições de saúde. Naquele momento, participavam de grupo de pesquisa e estavam responsáveis pela avaliação de condutores de automóveis com diagnóstico de doenças neurológicas como Doença de Parkinson (DP) e Acidente Vascular Encefálico (AVE). $\mathrm{Na}$ prática, estas profissionais atendem o público de adolescentes, adultos e idosos. TOA informou que a idade predominante de seus clientes compreende a faixa etária dos 16 à 25 anos e 75 à 90 anos.

No contexto americano, os terapeutas ocupacionais especialistas em reabilitação de condutores são referência para avaliação e treinamento na condução de automóvel ${ }^{2}$. Os terapeutas ocupacionais especialistas em reabilitação de condutores são encontrados em hospitais e centros de cuidado à saúde nos Estados Unidos ${ }^{13}$. Esses profissionais constituem programas e serviços de Terapia Ocupacional específicos para avaliação e reabilitação de condutores com mobilidade reduzida ${ }^{2,14,15}$.

Sobre conduzir, pode ser entendido como "controlar e mover, sob o seu próprio comando, um veículo ou o animal que o puxa, ou qualquer meio de transporte à sua disposição, como por exemplo, um carro [...]" (p.169) ${ }^{1}$. Este inclui o dirigir como meio de transporte, utilizando dentre vários meios de transporte, os veículos motorizados ${ }^{16}$. Sobre a dimensão dessa atividade e as exigências da integração do funcionamento físico com o sensorial e cognitivo necessários para que o condutor conduza o veículo de forma segura, considerando que a capacidade funcional sofre variações entre os indivíduos ${ }^{16}$. Para Classen et al. ${ }^{17}$, a direção segura é caracterizada pela redução de acidentes, erros, violações, lesões e mortes; desta forma, para dirigir com segurança, são importantes alguns eventos e interações entre esses eventos, os quais ocorrem na pessoa que dirige e no ambiente. Essa autora ${ }^{17}$ caracteriza a direção segura como o produto final de uma diversidade de eventos e interações que acontecem entre a pessoa, o automóvel e ambiente que é o trânsito. 
Fedeger AM, et al. Estratégias de avaliação de condutores de automóveis. Rev Ter Ocup Univ São Paulo. 2018 jan./abr.;29(1):50-5.

Para conduzir um automóvel, o condutor precisa das funções visuais, mentais e motoras com desempenho dinâmico e simultâneo. A atenção visual, campo de visão, visão periférica e movimentos de sacada são essenciais para a condução de um automóvel. A função executiva é a mais importante das funções mentais. A funcionalidade de movimentos finos da mão e diferentes tipos de preensão, como por exemplo, acionar a alavanca do pisca, agarrar e utilizar ambas as mãos no volante, controlar o câmbio de marchas, abrir e fechar a porta do veículo ${ }^{18}$.

Quanto ao raciocínio profissional do terapeuta ocupacional sobre a condução de automóvel, os autores detêm-se a descrever as demandas e habilidades para o desempenho da atividade e os fatores pessoais e contextuais relacionados a ela. Considerar o significado em ser condutor para o cliente requer a compreensão do grau de importância desta atividade no cotidiano de uma pessoa. Com o avanço da tecnologia de assistência, o terapeuta ocupacional participa do desenvolvimento e utilização de recursos adaptativos que facilitem o desempenho e minimizem o risco na condução de automóvel em pessoas com diferentes deficiências ${ }^{14,15,18}$.

Sobre os procedimentos técnicos no processo de reabilitação de condutores de automóveis, serão abordados o encaminhamento ao serviço, o processo de avaliação, resultados e recomendações realizados pelos terapeutas ocupacionais.

O encaminhamento para o serviço especializado de terapeutas ocupacionais reabilitadores de condutores ocorre por intermédio dos profissionais de saúde, principalmente os médicos, e também por terapeutas ocupacionais que em atendimento clínico em outros programas constatam risco para a condução de automóveis em decorrência de limitações físicas, neurológicos, cognitivas e visuais ${ }^{2}$.

A avaliação clínica realizada pelo terapeuta ocupacional ou qualquer outro membro da equipe de reabilitação e serve para identificar pontos fortes e deficiências relacionadas à condução de veículos; é feita uma análise sobre a "informação médica, dos remédios e seus efeitos colaterais, episódios de convulsões ou perda de consciência, estado de mobilidade, histórico social, histórico vocacional, histórico de motorista e propósito da avaliação" (p.218)2. A avaliação do condutor pelo terapeuta ocupacional tem tempo superior a 150 minutos, aproximadamente 3 horas, segundo as participantes da pesquisa.

Além disso, as participantes da pesquisa responderam que em relação às condições de saúde de seus clientes com mobilidade reduzida, observa-se o uso e/ou a necessidade de adaptação para a atividade, uso de prótese, medicação, acuidade visual e outras funções da visão, postura estática e o diagnóstico. A TOA acrescentou que está atenta a questões relacionadas à obesidade $\mathrm{e}$ acuidade auditiva do condutor.

Nos programas de avaliação e reabilitação de condutores, os terapeutas ocupacionais consideram o diagnóstico, sintomas, progressão e as condições de saúde do condutor, o desempenho apto em exames clínicos motores, cognitivos e visuais, em testes de simuladores de condução, em provas práticas na condução de automóvel em teste de rua e prova de estacionamento.

Sobre os exames clínicos, a TOA descreveu os protocolos utilizados para avaliar funções visuais, cognitivas e motoras dos condutores: Vision Screening e Useful Field of View, rastreio da acuidade visual; Trails A e B e Clock Draw Test, rastreio de habilidades cognitivas; e Rapid Pace Walk, rastreio de habilidades motoras. As profissionais responderam que nem todos os protocolos de avaliação utilizados para exame clínico de condutores são validados ou de uso específico pelo terapeuta ocupacional.

O veículo utilizado no teste prático possui duplo comando, ou seja, freios duplos, um espelho auxiliar no lado do profissional avaliador, bem como sinalização adequada do veículo escola. A prova prática na avaliação do condutor é de suma importância, uma vez que proporcionam a observação da condução no trânsito real, assim o condutor está exposto às diversas condições do ambiente, o que permite identificar comportamento e desempenho do condutor ${ }^{2,15,19,20}$.

A avaliação na prova prática é iniciada por uma atividade de pré-direção, ou seja, é verificada a mobilidade da pessoa até o veículo, a inserção/giro da chave na porta do veículo bem como a abertura desta; entrar e sair do carro, ajuste do banco e equipamentos (por exemplo: espelhos e cinto de segurança). Em seguida, ocorre a avaliação quanto ao posicionamento adequado, função dos membros superiores, estabilidade do tronco e a utilização dos controles primários, sendo o volante por primeiro, identificando se há necessidade de uso de dispositivos adaptativos (por exemplo: direção com uma só mão) ${ }^{2}$.

Posteriormente a avaliação contínua com os controles de acelerador e freio; esses, por sua vez, são avaliados quanto a necessidade de extensão para pedais, troca de lado, por exemplo, em uma amputação, em que o membro inferior direito está ausente, e não há como operar pedais padrão (acelerador), para tanto, adapta-se um pedal ao lado esquerdo para compensar tal condição, ou então, 
modificação dos pedais para dispositivos de controle manual, quando da não possibilidade de utilização dos membros inferiores, caso de pessoas com lesão medular abaixo de T1. A próxima etapa consiste em avaliar a ativação dos controles secundários do veículo (setas, buzina, limpadores de para-brisas), estes por sua vez podem ser colocados no controle manual ou ser ativados com o auxílio do cotovelo, quando for o caso ${ }^{2}$.

Após todos esses procedimentos, parte-se para a avaliação prática do dirigir, realizada nas vias, com duração aproximada de quarenta e cinco minutos, não podendo exceder o tempo de duas horas. A rota envolve locais que promovam condições variadas do trânsito e que tenha acostamento para garantir a segurança ${ }^{2}$. Além disso, os terapeutas ocupacionais podem utilizar simuladores para facilitar a tomada de decisão a respeito da aptidão da pessoa para conduzir automóveis ${ }^{17}$.

As recomendações dos terapeutas ocupacionais especialistas em reabilitação de condutores têm como objetivo melhorar o desempenho de condução veicular e auxiliar com que os condutores mantenham sua independência dirigindo pelo maior tempo possível. Treinamento de habilidades para melhorar desempenho, ajustes e adaptações no veículo como espelhos maiores e modificação no controle da direção são descritos como procedimentos do terapeuta ocupacional ${ }^{2,13-15,19,20}$.

Sobre os programas de treinamento para condutores, são atendimentos com programa de atividades individualizados a fim de restaurar habilidades motoras, amplitude de movimento/força; estimulam a prática de atividades físicas, como caminhadas, que promovem o bem-estar; técnicas para utilização de dispositivos e tecnologias, além promover a segurança no trânsito para prevenir lesões e mortes por acidentes no trânsito².

$\mathrm{Na}$ literatura constatou-se que os testes práticos na condução de automóveis são realizados para a concessão e para renovação da concessão da licença para conduzir, seja para pessoas com mobilidade reduzida ou pelo processo de envelhecimento ${ }^{2,8,13,14,16,18}$. As participantes da pesquisa afirmaram que, nos Estados Unidos da América, seu parecer técnico pode interferir na concessão do direito da carteira de habilitação americana.
Das recomendações do terapeuta ocupacional há quatro possibilidades para o condutor: cancelamento da permissão de dirigir; suspensão temporária; restrições para o desempenho da atividade e a continuidade do desempenho na atividade ${ }^{22}$. Em situações que a pessoa realmente não pode conduzir um veículo, os terapeutas ocupacionais especialistas em trânsito auxiliam o cliente a identificar formas alternativas de transporte através do serviço de consultoria ${ }^{20}$.

\section{CONSIDERAÇÕES FINAIS}

O estudo apresentou um novo campo de pratica profissional e descreveu estratégias de terapeutas ocupacionais norte-americanas na avaliação de condutores de automóveis com mobilidade reduzida. O conhecimento produzido com auxilio da revisão de literatura possibilitou a compreensão sobre o desempenho requerido para a condução de automóvel com ênfase em condutores com mobilidade reduzida, a importância no cotidiano de pessoas em ir e vir, o risco no desempenho desta AIVD e os aspectos da politica publica de trânsito que legitimam o direito de conduzir um automóvel. A literatura contém elementos norteadores para a observação na visita técnica e elaboração do questionário. O questionário, como material de pesquisa, foi eficaz na produção dos dados que descreveram a especificidade certificada e o raciocínio do profissional terapeuta ocupacional. A literatura e os dados produzidos na pesquisa de campo favoreceram a descrição sobre os delineamentos do profissional terapeuta ocupacional no rastreio clinico dos aspectos físicos, motores, visuais e cognitivos e teste de rua de condutores de automóveis. Em relação a especialidade do terapeuta ocupacional norte-americano para reabilitar condutores de automóveis e o processo de certificação descritos neste estudo precisarão de marcos regulatórios éticos e políticos para uma prática profissional no Brasil. As contribuições das participantes deste estudo possibilitaram a compreensão necessária da relação entre assistência (reabilitação) e pesquisa. Este estudo constitui-se em marco importante e de referencia para terapeutas ocupacionais e aponta horizontes para novas investigações.

Contribuições dos autores: AM Fedeger - idealizou o manuscrito; HAG Teive e $R$ Nickel - realizaram orientação na pesquisa.

Agradecimentos: Fundação Araucária (398/2012). Coordenação de Aperfeiçoamento de Pessoal de Nível Superior - CAPES (PDSE 99999.003844/2014-05). Instituto de Mobilidade e Participação - IMAP, Universidade da Flórida, Gainesville, Flórida, Estados Unidos da América. Western University of London, Ontario, Canada. Professora Doutora Scherrilene Classen. Bolsistas de Iniciação Científica: Scheila Lucindo. Ana Raquel Silva. 
Fedeger AM, et al. Estratégias de avaliação de condutores de automóveis. Rev Ter Ocup Univ São Paulo. 2018 jan./abr.;29(1):50-5.

\section{REFERÊNCIAS}

1. Organização Mundial da Saúde. CIF-CJ: Classificação Internacional de Funcionalidade, Incapacidade e Saúde: versão para Crianças e Jovens. São Paulo: Editora da Universidade de São Paulo; 2011.

2. Lillie SM. Transporte, mobilidade na comunidade e avaliação da capacidade de dirigir. In: Pedretti LW, Early MB. Terapia Ocupacional: capacidades práticas para disfunções físicas. São Paulo: Roca; 2004. p.212-24.

3. American Occupational Therapy Association. Occupational Therapy practice framework: domain \& process. 2nd ed. Am J Occup Ther. 2008;62(6):625-83. https://doi.org/10.18554/ rt.v3i2.150.

4. Associação Brasileira de Normas Técnicas (ABNT). Norma Brasileira NBR 9050. Acessibilidade a edificações , mobiliário, espaços e equipamentos urbanos. Rio de Janeiro: ABNT; 2004. Disponível em: http://www.pessoacomdeficiencia.gov.br/ app/sites/default/files/arquivos/\%5Bfield_generico_imagensfilefield-description\%5D_24.pdf.

5. Brasil. Lei $n^{\circ}$. 9503, de 23 de setembro de 1997. Institui o Código de Trânsito Brasileiro. 24 set 1997; 184. Seção 1. Disponível em: http://www2.camara.leg.br/legin/fed/lei/1997/lei-9503-23setembro-1997-372348-publicacaooriginal-1-pl.html.

6. World Health Organization. Global status report on road safety. Supporting a decade of action. Geneva; 2014.

7. Hyder AA, Vercino-Ortiz AI. BRICS: opportunities to improve road safety. 2014;92:423-8. http://dx.doi.org/10.2471/ BLT.13.132613.

8. Classen S. Special issue on older driver safety and community mobility. Am J Occup Ther. 2010;64(2):211-4. doi: 10.5014/ ajot.64.2.211.

9. Thiellen IP. Carteira Nacional de Habilitação (CNH) e o desempenho de condutores de automóveis com mobilidade reduzida. In: I Simpósio Internacional sobre Aptidão no Desempenho de Pessoas com Mobilidade Reduzida; Curitiba, PR, 21 mar. 2014. Mesa redonda. Evento de Extensão. Universidade Federal do Paraná. Curitiba, Paraná,2014.

10. Brasil. Resolução n ${ }^{\circ} .267$, de 15 de fevereiro de 2008. Dispõe sobre o exame de aptidão física e mental, a avaliação psicológica e o credenciamento das entidades públicas e privadas. Departamento Nacional de Trânsito. Disponível em: http:// www.denatran.gov.br/download/Resolucoes/RESOLUCAO CONTRAN_267.pdf.

11. Brasil. Lei $n^{\circ}$. 12302, de 2 de agosto de 2010. Regulamenta o exercício da profissão de Instrutor de Trânsito. 3 ago 2010.
Seção 1. Disponível em: http://www.planalto.gov.br/ccivil_03/_ Ato2007-2010/2010/Lei/L12302.htm.

12. Driving and community mobility. Am J Occup Ther. 2010;64 (6 Suppl):S112-S124. doi:10.5014/ajot.2010.64S112.

13. Posse MC. Promoting older driver safety: impact of driving rehabilitation specialist recommendations on older adults' driving performance [thesis]. Flórida: University of Florida; 2008. Available from: http://etd.fcla.edu/UF/UFE0022543/ posse_m.pdf.

14. Stav W, Monahan M. The occupational therapy role in driving and community mobility across the lifespan. American Occupational Therapy Association. Occupational therapy practice framework: Domain and process. Am J Occup Ther. 2002;56(6):609-39. Available from: http://web1.ctaa.org/ webmodules/webarticles/articlefiles/Community_Mobility.pdf

15. Pierce S. Restauração da competência na mobilidade. In: Trombly CA, Radomski MV. Terapia ocupacional para disfunções físicas. 5a ed. São Paulo: Editora Santos; 2005. p. 685-92.

16. Finn J, Gross M, Hunt L, McCarthy D, Pierce S, Redepenning $\mathrm{S}$, et al. Driving Evaluation and retraining programs: A report of good practices. Betesta: AOTA Press; 2004.

17. Classen S. Special issue on older driver safety and community mobility. Am J Occup Ther. 2010;64(2):211-4. doi: 10.5014/ ajot.64.2.211.

18. Justiss MD. Development of a behind-the-wheel driving performance assessment for older adults [Thesis]. Florida: University Florida; 2005. Available from: http://etd.fcla.edu/ UF/UFE0013121/justiss_m.pdf.

19. Wang CC, Kosinski CJ, Schwartzberg JG, Shanklin AV. American Medical Association. Physician's guide to assessing and counseling older drivers. Washington: National Highway Traffic Safety Administration; 2003. Available from: http:// www.nhtsa.gov/people/injury/olddrive/physician_guide/ physiciansguide.pdf.

20. American Occupational Therapy Association. Occupational therapy: skills for the job of living. Tips for living. Keeping Older Drivers Safe. 2004.

21. Unsworth C. Using social judgment theory to study occupational therapist's use of information when making driver licensing recommendations for older and functionally impaired adults. Am J Occup Ther. 2007;61(5):493-502. Available from: https:// ajot.aota.org/article.aspx?articleid=1866983.

Recebido em: 21.11.17

Aceito em: 11.06.18 